HEPATOLOGY

\title{
MicroRNA profile before and after antiviral therapy in liver transplant recipients for hepatitis $C$ virus cirrhosis
}

\author{
Fanni Gelley, ${ }^{\dagger}$ Gergely Zadori, ${ }^{\dagger}$ Balazs Nemes, ${ }^{\dagger}$ Matteo Fassan, ${ }^{\S}$ Gabor Lendvai, ${ }^{*}$ Eniko Sarvary, ${ }^{\dagger}$ \\ Attila Doros, ${ }^{\dagger}$ Zsuzsanna Gerlei, ${ }^{\dagger}$ Peter Nagy, ${ }^{\ddagger}$ Zsuzsa Schaff* and Andras Kiss* \\ *2nd Department of Pathology, ${ }^{\dagger}$ Department of Transplantation and Surgery, and ${ }^{\ddagger} 1$ st Department of Pathology, Semmelweis University, \\ Budapest, Hungary; and ${ }^{\S}$ ARC-NET Research Centre, University of Verona, Verona, Italy
}

\begin{abstract}
Key words
$\mathrm{HCV}$ receptors, HCV, liver transplantation, microRNA, SVR.
\end{abstract}

Accepted for publication 2 August 2013.

\section{Correspondence}

Dr András Kiss, 2nd Department of Pathology, Semmelweis University, 1091 Budapest, Üllői út 93. Hungary. Email:

kiss.andras@med.semmelweis-univ.hu

\begin{abstract}
Background and Aim: Management of hepatitis C virus (HCV) recurrence is a major challenge after liver transplantation. Significant dysregulated expression of HCV receptors (i.e. claudin-1, occludin, tetraspanin CD81, scavenger receptor type B1) has been shown recently during $\mathrm{HCV}$ infection. This might facilitate hepatocytic entry and reinfection of HCV. MicroRNAs (miRs) play role in the regulation of gene expression. We aimed to characterize miR expression profiles related to $\mathrm{HCV}$ infection and antiviral therapy in adult liver transplant recipients, with special emphasis on miRs predicted to target HCV receptors.

Methods: Twenty-eight adult liver transplant recipients were enrolled in the study. Paired biopsies were obtained at the time of HCV recurrence and at the end of antiviral treatment. MiRs for HCV receptors were selected using target prediction software. Expression levels of miR-21, miR-23a miR-34a, miR-96, miR-99a*, miR-122, miR-125b, miR-181a-2*, miR-194, miR-195, miR-217, miR-221, and miR-224 were determined by reverse transcription-quantitative polymerase chain reaction.

Results: miR-99a* and miR-224 expressions were increased in HCV recurrence samples, while miR-21 and miR-194 were decreased in comparison to normal liver tissue. Increased expressions of miR-221, miR-224, and miR-217 were observed in samples taken after antiviral therapy when compared with $\mathrm{HCV}$ recurrence samples. High HCV titer at recurrence was associated with higher level of miR-122.

Conclusions: Samples at recurrence of HCV and after antiviral therapy revealed distinct HCV-related miR expression profiles, with significant dysregulation of those miRNAs potentially targeting mRNAs of HCV receptors. In particular, miR-194 and miR-21 might be involved in the regulation of $\mathrm{HCV}$ receptor proteins' expression during $\mathrm{HCV}$ infection and antiviral therapy.
\end{abstract}

\section{Introduction}

Hepatitis C virus (HCV) infection-related chronic liver disease is a significant health problem worldwide and is the leading indication for orthotopic liver transplantation (OLT). ${ }^{1,2} \mathrm{HCV}$ recurrence after transplantation is universal and represents a major therapeutic challenge as current standard therapy against $\mathrm{HCV}$ infection is limited to the combination of pegylated interferon- $\alpha$ and ribavirin (IFN/RBV). However, the therapy has considerable side-effects, and the response rate is less than ideal. $^{3}$

Several cellular factors are known to be involved in the hepatocellular entry mechanism of $\mathrm{HCV}^{4}$ Tight-junction (TJ) proteins claudin-1 (CLDN1) and occludin (OCLN), CD81, scavenger receptor class B type 1 (SCARB1) have been reported to be part of the internalization complex and are essential for HCV entry both in vitro and in vivo. ${ }^{2,5-7}$

Previous studies have shown discrepancies between messenger RNA (mRNA) and protein expression levels of these receptors in $\mathrm{HCV}$ infected patients, suggesting therefore the role of microRNAs (miRs) in the modulation of gene expression. ${ }^{2,4,5}$

MiRs are small endogenous non-coding $~ 22$ nucleotide long RNAs that regulate gene expression at posttranscriptional level. MiRs induce degradation of the mRNA or suppression of protein translation upon binding to the $3^{\prime}$ untranslated regions (UTRs) of an mRNA. ${ }^{8,9}$ One microRNA sequence might interact with several mRNAs. Therefore, miRs exert negative regulation on mRNA and finally on protein expression. They are essential for maintenance of cellular homeostasis and normal function, serving as key regulators of various biological processes 
Table 1 Baseline characteristics of patients

\begin{tabular}{lc}
\hline Age & $50 \pm 5.4$ \\
Gender (male/female) & $18 / 10$ \\
MELD score & $13.5 \pm 5$ \\
HCV RNA $\left(10^{6} / \mathrm{mL}\right)$ at recurrence & $8.2 \pm 10.7$ \\
HCV RNA $\left(10^{6} / \mathrm{mL}\right)$ after therapy & $1.9 \pm 4.5$ \\
Tacrolimus based IS & $20(71.4 \%)$ \\
Cyclosporin A IS & $8(28.6 \%)$ \\
HAl (Knodell score) 1-8 & \\
At recurrence 1-4/5-8 & $21 / 7$ \\
After therapy 1-4/5-8 & $27 / 1$ \\
Fibrosis score 0-6 & \\
At recurrence 0-1/2-3/4-6 & $21 / 6 / 1$ \\
After therapy 0-1/2-3/4-6 & $20 / 5 / 3$ \\
Therapy response & \\
NR & $22(78.6 \%)$ \\
SVR & $6(21.4 \%)$ \\
\hline
\end{tabular}

$\mathrm{HAI}$, histology activity index; $\mathrm{HCV}$, hepatitis $\mathrm{C}$ virus; IS, immunosuppression; MELD, model for end-stage liver disease; NR, non-responder; SVR, sustained virological response.

including cellular stress, steatosis, proliferation, differentiation, and apoptosis. ${ }^{9-11}$

Dysregulated expression of miRs might play role in the development of many diseases including viral infections and malignancies. ${ }^{12-14}$ MiRs also participate in the control of $\mathrm{HCV}$ infection as well as in the IFN pathway via several mechanisms. ${ }^{3,15}$ The liver-specific microRNA, miR-122, is known as a positive cofactor in $\mathrm{HCV}$ replication cycle $^{16}$ and has been found to be downregulated by $\alpha / \beta$ IFNs. ${ }^{17}$ Besides, IFN- $\beta$ has been described as modulator of the expression of several miRs having sequence-predicted targets within the HCV genomic RNA, and some of these miRs were shown to actively inhibit $\mathrm{HCV}$ replication. ${ }^{17,18}$ In addition, the hepatic microRNA expression pattern existing in chronic $\mathrm{HCV}$-infected patients before antiviral therapy was shown to be associated with therapy response. $^{3}$

The aim of the current study was to evaluate the expression of miRs, which either have one of the HCV receptors among their target mRNAs according to target prediction software (such as miR-21, miR-34a, miR-96, miR-125b, miR-194, miR-195, and miR-224), or which may play role in HCV infection or in antiviral treatment response (such as miR-23a, miR-99a*, miR-122, miR181a-2*, miR-217, and miR-221) in liver transplant recipients.

\section{Materials and methods}

Patients and sample preparation. A total of 28 liver needle biopsies of HCV-positive adult liver transplanted patients were included. These selection criteria were applied: (i) HCVrelated cirrhosis and subsequent liver transplantation; (ii) histologically proved recurrence followed by completed 12 months uninterrupted antiviral therapy; (iii) 6 months follow up after antiviral therapy.

Baseline characteristics of patients are summarized in Table 1. In the majority of patients $(n=22,78.6 \%), \mathrm{HCV}$ recurred within the first postoperative year. Liver biopsies were taken twice: at the time when $\mathrm{HCV}$ recurrence was observed following liver transplantation and after the end of antiviral therapy. Normal liver samples $(n=13)$ were obtained from deceased donors during organ receiving, just before ligation of the abdominal aorta and reperfusion. These donor livers were used for transplantation before the start of this project. Liver samples were fixed in $10 \%$ buffered formalin and embedded in paraffin. HAI (histology activity index, modified ISHAK score /0-18/) and fibrosis score /0-6/ were determined for histological grading and staging of liver specimens. The study followed the ethical guidelines of the 1975 Declaration of Helsinki. Informed consent was obtained from all patients included in the study.

Treatment protocol and definitions of therapy response. All selected patients received the combination of IFN/RBV for 12 months without interruption. Patients with good renal function received pegylated IFN $2 b$, while patients with impaired renal function were treated with pegylated IFN 2a. No additional treatment was applied. Six patients $(21 \%)$ achieved sustained viral response (SVR: HCV was undetectable in the sera using reverse transcription-polymerase chain reaction [RT-PCR] 6 months following the completion of IFN/RBV therapy). Patients were defined as being non-responders (NR) if their sera were positive for HCV RNA (22 patients). All patients had HCV genotype 1b infection. There were no significant differences in patient gender or age between the NR and SVR groups.

MicroRNA target prediction. In silico identification of miRs that may bind to any mRNAs of HCV receptors CLDN1, $O C L N$, SCARB1, and CD81 was performed using microRNA.org (http://www.microrna.org) target prediction database and software application developed by Tömböl and coworkers. ${ }^{19}$ The latter is capable of merging three target prediction databases such as TargetScan 6.0 (http://www.targetscan.org), PicTar (http://pictar.mdc-berlin.de), and MicroCosm Targets Version 5 (http://www.ebi.ac.uk/enright-srv/microcosm/htdocs/targets/v5/). The database search resulted in about 550 specific miRs, from which there were 39 (CLDN1), 13 (OCLN), 1 (miR-194; CD81), and 8 (SCARB1) miRs commonly present in the database lists for the four mRNAs, respectively. Finally, the microRNA lists were narrowed down by either selecting the consensus sequences of all three databases or the sequences possibly targeting several mRNAs of different $\mathrm{HCV}$ receptor types, or mRNAs connected to HCV hepatitis according to the literature. The following miRs were selected to modulate $\mathrm{HCV}$ receptor expression by target prediction and based on sequence homology: CLDN1: 21, 34a, 96, 194, and 195; OCLN: 122, 194, and 224; CD81: 23a, 125b, and 194; SCARB1: 96, 99a*, 125b, and 195 (Letters in bold indicate consensus in all three databases).

RNA extraction and RT-quantitative PCR. RNA was isolated using RNeasy FFPE Mini Kit (QIAGEN, Hilden, Germany) according to the manufacturer's instructions with modification for copurification of miRNA, then stored at $-80^{\circ} \mathrm{C}$. Contaminating genomic DNA was removed using Turbo DNase digestion (Ambion Life Technologies, Carlsbad, CA, USA) according to the manufacturer's instructions. 
MicroRNA expression was determined applying the following TaqMan MicroRNA Assays (Applied Biosystems, Carlsbad, CA, USA): miR-21 (ID:000397), miR-23a (ID:000399), miR-34a (ID:000426), miR-96 (ID:000186), miR-99a* (ID:002141), miR-122 (ID:002245), miR-125b (ID:000449), miR-181a-2* (ID:002317), miR-194 (ID:000493), miR-195 (ID:000494), miR-217 (ID:002337), miR-221 (ID000524), and miR-224 (ID:002099). RT was performed using TaqMan MicroRNA Reverse Transcription Kit (Applied Biosystems) according to the manufacturer's instructions in $7.5 \mu \mathrm{L}$ final volume containing $10 \mathrm{ng}$ total RNA. TaqMan Universal PCR Master Mix No AmpErase UNG (Applied Biosystems) was applied for real-time PCR, also carried out according to the manufacturer's instructions, in $10 \mu \mathrm{L}$ final volume containing $0.65 \mu \mathrm{L}$ RT product. The reaction was run on an ABI PRISM 7000 system (Applied Biosystems). The samples were measured in duplicates. Relative expression level in samples was determined by $2^{\Delta \mathrm{Cq}}$ method using the mean $\mathrm{Cq}$ value of miR-23a and miR-34a as reference. These reference miRs showing the overall least variation among samples were selected using NormFinder ${ }^{20}$ as U6 snRNA (ID:001973) proved to be highly variable in the samples.

Statistical analysis. SPSS 15. version (SPSS, Inc., Chicago, IL, USA) was used for the statistical analysis. Continuous variables are shown as mean values and standard deviations. Student's $t$-test, ANOva test with Scheefe and Bonferroni post hoc tests, as well as Mann-Whitney $U$-test were utilized for univariate analyses, after examining population homogeneity of the variables (Levene test). The ANOVA method was used to compare microRNA expression before and after IFN therapy, when therapy response (SVR vs NR) was taken into account. The connections between continuous variables were evaluated by correlation analysis, using Pearson correlation coefficient. $P<0.05$ was considered significant.

\section{Results}

Baseline characteristics of patients are shown in Table 1. Examination of the clinical data revealed that post-treatment viral load decreased significantly only in the SVR group when compared with pretreatment levels $\left(12 \times 10^{6} / \mathrm{mL} \pm 15.7\right.$ vs $0 / \mathrm{mL}, P=$ $0.003)$. HAI decreased in both NR (3.74 \pm 1 vs $1.6 \pm 1$, $P<0.0001)$ and SVR groups $(4 \pm 1$ vs $1 \pm 1.3, P=0.002)$ after administering IFN/RBV therapy. After finishing antiviral therapy fibrosis score was analyzed, and no correlation was found between fibrosis being above or below the median (fibrosis score 1) and the investigated miRs. High viral load (above median $3.4 \times 10^{6} / \mathrm{mL}$ ) at the time of $\mathrm{HCV}$ recurrence was associated with higher expression level of miR-122 (relative expression below median $132.8 \pm 79$ vs above median $2252.6 \pm 2663, P=0.025)$. The expression level of miR-221 negatively correlated with HAI $(r=-0.313, P=0.036)$.

The transcriptional levels of miR-99a* and miR-224 were significantly increased, while miR-21 and miR-194 were decreased in liver samples obtained at HCV recurrence, as compared with the levels measured in normal liver tissue. Results are shown in Table 2a and Figure 1a.
To examine whether IFN/RBV therapy has impact on microRNA expression after OLT, we compared paired liver samples of patients obtained before and after antiviral treatment. In comparison with pretreatment expression levels, increased expressions were found for miR-221, miR-224, and miR-217 in samples taken after administration of antiviral treatment (Table $2 b$ and Fig. 1b.).

MicroRNA expression levels were also investigated in relation to therapy response. Because of the fact that only SVR is associated with long-term clinical improvement, we focused on this patient group $(n=6 ; 21 \%)$. SVR patients showed significantly increased miR-96, miR-99a*, miR-122, miR-181a-2*, miR-217, and miR-221 expression levels in comparison with non-responders at the end of aniviral therapy (Table 2c and Fig. 1c.). Among SVR patients, a significant upregulation of miR-221 (0.664 \pm 0.82 before $v s \quad 6.728 \pm 10$ after, $P=0.017)$ and miR-122 (1557.6 \pm 3005 before vs $11103.8 \pm 16666$ after, $P=0.038$ ) was observed at the end of the treatment. The pretreatment microRNA profile was not predictive for the success of antiviral therapy.

\section{Discussion}

The recurrence of HCV is related to lower survival rates after liver transplantation. ${ }^{21}$ Moreover, disease progression is accelerated after OLT because of the immunsuppressed status of the patients. ${ }^{22}$ Current standard IFN/RBV therapy against HCV is known to be effective in only $50 \%$ of patients infected with the prevalent genotype $1 .{ }^{23} \mathrm{HCV}$ recurrence in liver-transplanted patients is therefore a suitable model to study the pathomechanism of $\mathrm{HCV}$ reinfection and the effect and outcome of antiviral therapy.

This is the first study examining the expression of miRs targeting $\mathrm{HCV}$ receptors in liver transplant patients due to chronic HCVinduced liver failure. The miRs were selected on the basis of in silico target prediction, focusing on HCV receptors.

After non-specific attachment to the cell surface molecules such as low-density lipoprotein receptors and glycosaminoglicans, HCV particles are consecutively bound to a complex formed by SCARB-1 and CD81. Virus associated with CD81 would then be transferred into TJs, where HCV would interact with CLDN-1 and OCLN to enter the cell. ${ }^{2}$ The expression of CLDN-1 and OCLN proteins is increased in $\mathrm{HCV}$-infected liver compared with normal liver tissue ${ }^{4,5}$ and in HCV recurrence after OLT. ${ }^{2}$ However, there was no correlation between mRNA and protein levels of these receptors. ${ }^{2}$ Similarly, in a recent paper by Nakamuta and colleagues, different mRNA and protein expressional levels were demonstrated for CLDN-1 and OCLN in HCV-infected livers. ${ }^{4}$ These data suggest that CLDN-1 and OCLN expression may be significantly influenced at post-transcriptional level in the presence of $\mathrm{HCV}$.

Dysregulated microRNA expression patterns have been reported in many human diseases, such as various types of cancers, as well as metabolic, infectious, chronic inflammatory, and autoimmune diseases. ${ }^{24,25}$

Regarding HCV infection, the liver-specific miR-122 has been reported to enhance $\mathrm{HCV}$ replication in human hepatoma cells by binding to the $5^{\prime}$ UTR of $H C V$, and sequestration of miR-122 by antisense oligonucleotide decreased HCV replication and translation in vitro ${ }^{16,23,26-28}$ and in vivo. ${ }^{29,30}$ However, no ${ }^{31}$ or only weak positive correlation ${ }^{13}$ could be found between hepatic miR-122 and 
Table 2 MicroRNA expressions

\begin{tabular}{|c|c|c|c|c|}
\hline \multicolumn{5}{|c|}{ A. MicroRNA expression at HCV recurrence compared with normal liver tissue } \\
\hline \multirow[t]{2}{*}{ MicroRNA } & \multicolumn{2}{|l|}{ Relative expression } & \multirow[t]{2}{*}{ Mean ratio $(r / d)$} & \multirow[t]{2}{*}{$P$ value } \\
\hline & Donor samples & HCV-infected samples & & \\
\hline miR-21 & $198.7 \pm 89.6$ & $46.8 \pm 30.6$ & 0.23 & $<0.001$ \\
\hline miR-96 & $0.0045 \pm 0.0044$ & $0.0018 \pm 0.0016$ & 0.4 & NS \\
\hline miR-99a* & $0.25 \pm 0.13$ & $0.82 \pm 0.74$ & 3.28 & 0.002 \\
\hline miR-122 & $2005 \pm 1586$ & $1280 \pm 1910$ & 0.63 & NS \\
\hline miR-125b & $28.7 \pm 13.7$ & $35.5 \pm 35.3$ & 1.18 & NS \\
\hline miR-181a-2* & $0.15 \pm 0.15$ & $0.19 \pm 0.16$ & 1.2 & NS \\
\hline miR-194 & $157.3 \pm 89.8$ & $37.6 \pm 35.2$ & 0.24 & $<0.001$ \\
\hline miR-195 & $15 \pm 8$ & $11.2 \pm 10.8$ & 0.74 & NS \\
\hline $\operatorname{miR}-217$ & $0.018 \pm 0.01$ & $0.013 \pm 0.02$ & 0.72 & NS \\
\hline miR-221 & $0.64 \pm 0.48$ & $0.34 \pm 0.35$ & 0.53 & NS \\
\hline \multirow[t]{2}{*}{$\operatorname{miR}-224$} & $0.12 \pm 0.1$ & $0.24 \pm 0.23$ & 2.18 & 0.039 \\
\hline & \multicolumn{3}{|c|}{ B. MicroRNA expression before and after antiviral therapy } & \\
\hline \multirow[t]{2}{*}{ MicroRNA } & \multicolumn{2}{|c|}{ Relative expression } & \multirow[t]{2}{*}{ Mean ratio (a/b) } & \multirow[t]{2}{*}{$P$ value } \\
\hline & Before & After & & \\
\hline miR-21 & $46.8 \pm 25.6$ & $32.17 \pm 37.3$ & 0.68 & NS \\
\hline miR-96 & $0.0018 \pm 0.0018$ & $0.008 \pm 0.007$ & 4.4 & NS \\
\hline miR-99a* & $0.8 \pm 0.64$ & $1.1 \pm 1.3$ & 1.3 & NS \\
\hline miR-122 & $1280 \pm 1717$ & $1219 \pm 1914$ & 0.9 & NS \\
\hline miR-125b & $35.5 \pm 36.8$ & $43.8 \pm 42.3$ & 1.2 & NS \\
\hline miR-181a-2* & $0.19 \pm 0.18$ & $0.26 \pm 0.27$ & 1.3 & NS \\
\hline miR-194 & $37.6 \pm 36.4$ & $46 \pm 38.2$ & 1.2 & NS \\
\hline miR-195 & $11.2 \pm 11$ & $7.86 \pm 9.9$ & 0.7 & NS \\
\hline $\operatorname{miR}-217$ & $0.013 \pm 0.1$ & $0.023 \pm 0.02$ & 1.77 & 0.042 \\
\hline $\operatorname{miR}-221$ & $0.34 \pm 0.36$ & $0.73 \pm 0.43$ & 2.1 & 0.006 \\
\hline \multirow[t]{2}{*}{ miR-224 } & $0.24 \pm 0.2$ & $0.72 \pm 0.88$ & 3 & 0.022 \\
\hline & \multicolumn{3}{|c|}{ C. MicroRNA expression after antiviral therapy in SVR and NR group } & \multirow{3}{*}{$P$ value } \\
\hline \multirow[t]{2}{*}{ MicroRNA } & \multirow{2}{*}{\multicolumn{2}{|c|}{$\begin{array}{l}\text { Relative expression } \\
\text { Before After }\end{array}$}} & \multirow[t]{2}{*}{ Mean ratio (a/b) } & \\
\hline & & & & \\
\hline miR-21 & $30.8 \pm 36$ & $70.1 \pm 110$ & 2.3 & NS \\
\hline miR-96 & $0.006 \pm 0.005$ & $0.12 \pm 0.18$ & 20 & 0.01 \\
\hline miR-99a* & $0.57 \pm 0.57$ & $3.14 \pm 4.7$ & 5.5 & 0.034 \\
\hline miR-122 & $1565.3 \pm 2039$ & $11103.8 \pm 16666$ & 7 & 0.014 \\
\hline miR-125b & $39.2 \pm 43.4$ & $14.4 \pm 12.4$ & 0.36 & NS \\
\hline miR-181a-2* & $0.14 \pm 0.19$ & $0.63 \pm 0.76$ & 4.5 & 0.017 \\
\hline miR-194 & $35.7 \pm 27.4$ & $50.8 \pm 57.2$ & 1.4 & NS \\
\hline miR-195 & $6.4 \pm 7.2$ & $22.9 \pm 49.5$ & 3.6 & NS \\
\hline miR-217 & $0.024 \pm 0.025$ & $3.7 \pm 7.3$ & 185 & 0.045 \\
\hline miR-221 & $0.8 \pm 0.6$ & $6.72 \pm 10$ & 8.4 & 0.003 \\
\hline miR-224 & $0.72 \pm 0.8$ & $0.51 \pm 0.3$ & 0.7 & NS \\
\hline
\end{tabular}

HCV, hepatitis C virus; miR, microRNA; NR, non-responder; NS, non-significant; SVR, sustained virological response.

serum HCV load, while no correlation could be observed between hepatic miR-122 and hepatic HCV load. ${ }^{13,31}$ Further, miR-122 was downregulated in acute HCV infection in human hepatoma cells at day 4 post-infection, ${ }^{9}$ and IFN- $\beta$ reduced the expression of miR-122. ${ }^{17}$ This indicates that miR-122 may be affected by the consequences of $\mathrm{HCV}$ infection and IFN treatment. In the present study, higher expression level of miR-122 was associated with higher viral load in patient sera; however, no significant difference was found in hepatic miR-122 expression at the time of $\mathrm{HCV}$ recurrence compared with normal liver tissue.
Applying the same comparison, the expression levels of miR-21 and miR-194 were decreased, whereas those of miR-99a* and miR-224 were increased upon HCV reactivation when compared with the normal hepatic expression of these miRs. miR-21 and miR-194 were found to influence $C L D N-1$ mRNA expression, while miR-99a* might control $S C A R B-1$ expression and miR-224 might modulate mRNA of $O C L N$. In silico sequence comparison also suggests the binding of miR-194 to mRNAs of OCLN and CD81. Therefore, the observed expressional increase of CLDN-1 and OCLN ${ }^{4,5}$ might be caused by the decrease of miR-194 and 
(a)

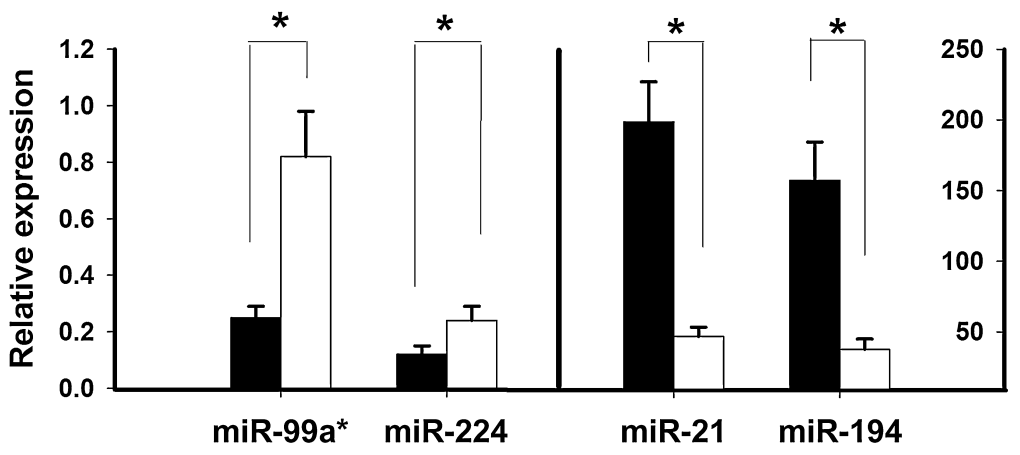

Figure 1 Hepatic expression of microRNAs in normal liver tissue and in liver transplanted patients at hepatitis $\mathrm{C}$ virus $(\mathrm{HCV})$ recurrence and after interferon/ribavirin therapy. Variables are shown as mean values and standard error of means (SEM). * symbolizes significant difference $(P<0.05)$. (a) Relative expression rate of microRNAs at HCV recurrence in livertransplanted patients and in normal liver tissue. Black bars represent normal donor samples $(n=13)$, and white bars represent biopsies obtained at HCV recurrence $(n=28)$. (b) Relative expression rate of microRNAs before and after interferon/ribavirin therapy in liver-transplanted patients. Black bars represent biopsies obtained before antiviral therapy, and white bars represent samples taken after antiviral therapy. (c) Relative expression rate of microRNAs after interferon/ribavirin therapy in sustained viral response (SVR) and non-responder (NR) groups. Black bars represent biopsies of nonresponder patients, and white bars show biopsies of patients who reached SVR after antiviral treatment. miR, microRNA.F (b)

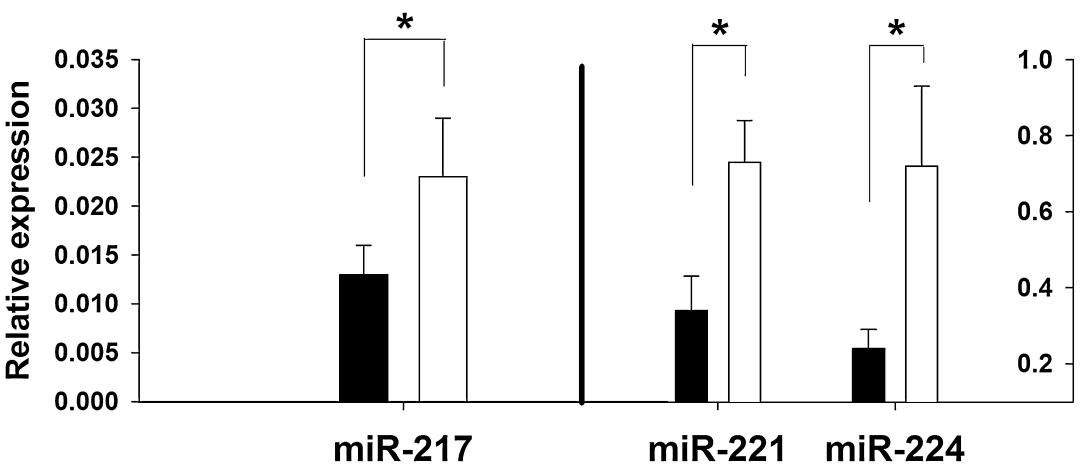

(c)

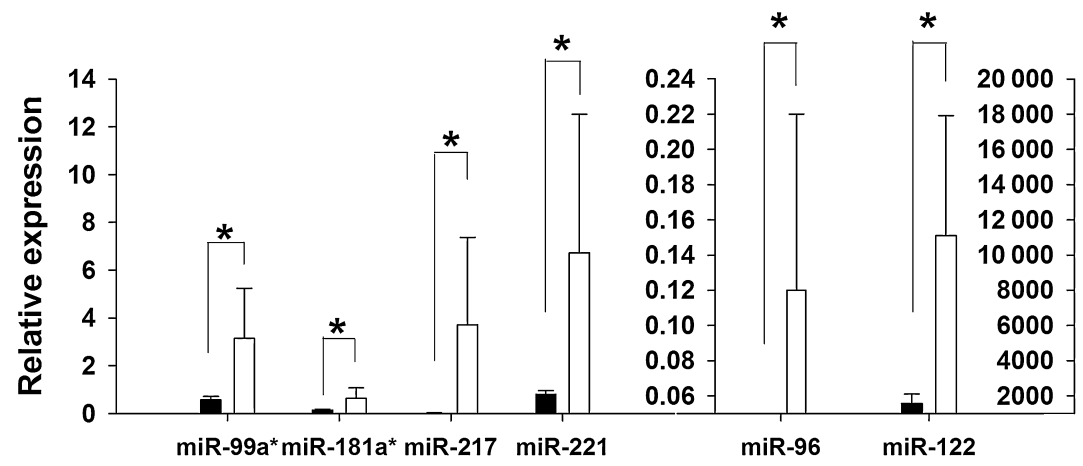

miR-21 expressions upon HCV infection. This would represent in vivo function of these miRs in the post-transcriptional regulation of $\mathrm{HCV}$ receptors.

The high expression of miR-194 in normal liver tissue has been known for a long time. ${ }^{32}$ miR-194 plays a role in the regulation of hepatic stellate cell activation during fibrogenesis ${ }^{33}$ and suppresses $\mathrm{N}$-cadherin expression, leading to inhibition of cell migration, adhesion, and metastasis of hepatocellular carcinoma (HCC) cells. ${ }^{32}$ miR-21 has been previously identified as an "onco-miR" because of its abberant expression in multiple malignancies including breast cancer, colon, and HCCs. ${ }^{34}$ Interestingly, our dataset showed decreased HCV recurrence-associated expression of miR-21. Certain other changes, however, such as elevated miR-224 expression found in our study during HCV recurrence, were also detected in HCCs induced by HCV infection. ${ }^{35}$

Upregulated miR-224 was found in HCC cell lines and HCCs. Recently, Scisciani and colleagues demonstrated that lipopolysaccharide, lymphotoxin- $\alpha$, and tumor necrosis factor- $\alpha$ inflammatory pathways activated miR-224 expression. In addition, these authors identified $p 65 / N F \kappa B$ as a direct transcriptional regulator of miR-224 expression. ${ }^{36}$ In our study, increased expression of miR224 upon HCV reactivation is in accordance with the findings of Scisciani and colleagues, ${ }^{36}$ as $\mathrm{NF \kappa B}$-dependent inflammatory pathway is a key process during $\mathrm{HCV}$ infection. ${ }^{37}$

Increased expressions of miR-221, miR-224, and miR-217 were observed in samples taken after administration of IFN/RBV treatment as compared with the pretreatment samples. miR-224 was able to recognize $O C L N$ as a target mRNA. miR-221 expression is dysregulated in $\mathrm{HCC}^{38}$ and is suggested to be affected by HCV and IFN. Liu and colleagues demonstrated that miR-221 was downregulated after HCV exposure, and the gain of miR-221 enhanced HCV RNA abundance in a dynamic in vitro $\mathrm{HCV}$ infection system. ${ }^{9}$ Zhang and colleagues knocked down miR-221 and miR-222 in glioblastoma cells and reported that IFN- $\alpha$ was the most significantly affected signaling pathway. ${ }^{39}$ Interestingly, we found that miR-221 expression level negatively correlated with HAI. miR-217 was previously shown to potentially predict therapy response in chronic $\mathrm{HCV}$-infected 
patients ${ }^{40}$ and was found to be related to tumor differentiation as well. ${ }^{14}$

We observed relevant alterations of microRNA expressions in the SVR group, whereas microRNA expression levels remained stable in non-responders after IFN/RBV treatment in comparison with pretreatment levels. Partial responders following completed antiviral therapy were only three patients; therefore, these patients were analyzed together within the NR group. It was recently revealed that hepatic microRNA expression could be associated with drug response ${ }^{3}$; however, our study did not reveal a predictive value of pretreatment microRNA levels, including miR-122, for the success of IFN/RBV treatment. Previously, Sarasin-Filipowicz and colleagues demonstrated markedly decreased pretreatment miR-122 levels in patients who had no virological response during later IFN therapy. ${ }^{31}$ Estrabaud and colleagues reported deregulated miR-99a*, miR-181a-2*, miR23a, and miR-217 in non-responders compared with patients with later SVR. ${ }^{40}$ In contrast, our study revealed upregulated miR-96, miR-99a*, miR-122, miR-181a-2*, miR-217, and miR221 expressions after IFN/RBV treatment in the SVR group when compared with the NR group. In addition, following antiviral treatment, miR-221 and miR-122 levels were higher in SVR when compared with pretreatment levels. This suggests that antiviral therapy restored miR-122 expression in SVR patients. miR-122 is considered a differentiation and homeostatis marker in hepatocytes. ${ }^{41}$ Given that miR-221 is suggested to be oncogenic in HCC and miR-122 is crucial for $\mathrm{HCV}$ proliferation, this phenomenon further strengthens the complex way how miRs take action and rules out simple mechanistic explanations. There were no statistical differences in therapy response or microRNA expression between the patient groups being either on tacrolimus or cyclosporin based immunosuppression.

The interpretation of our study has some limitations. By the clinical point of view, all of the considered patients belonged to genotype $1 / b$; therefore, we might draw conclusions regarding only to this patient group. Another point is that the normal liver samples did not derive from the same transplanted liver because the transplantation protocol of our institute did not contain zero time point liver biopsy in the time frame of the study. The normal liver samples were received from other donor livers harvested before the start of this project. However, the donor selection criteria for the transplantation remained the same.

In conclusion, we demonstrated that $\mathrm{HCV}$ recurrence and antiviral therapy is associated with altered hepatic expression of miRs including those microRNAs, which potentially target mRNAs of HCV receptors. Our data suggest that particularly miR-194 and miR-21 may be involved in expressional regulation of $\mathrm{HCV}$ receptor proteins during $\mathrm{HCV}$ infection and antiviral therapy.

\section{Acknowledgments}

Authors would like to thank Elvira Kale-Rigo for her help in editing and correcting the manuscript, and Mrs. Magdolna Pekár for her technical assistance.

This work was supported by grant \#OTKA K101435 from the Hungarian National Scientific Research Foundation.

\section{References}

1 Khapra AP, Agarwal K, Fiel MI et al. Impact of donor age on survival and fibrosis progression in patients with hepatitis $\mathrm{C}$ undergoing liver transplantation using $\mathrm{HCV}+$ allografts.

Liver Transpl. 2006; 12: 1496-503.

2 Mensa L, Crespo G, Gastinger MJ et al. Hepatitis C virus receptors claudin-1 and occludin after liver transplantation and influence on early viral kinetics. Hepatology 2011; 53: 1436-45.

3 Murakami Y, Tanaka M, Toyoda $\mathrm{H}$ et al. Hepatic microRNA expression is associated with the response to interferon treatment of chronic hepatitis C. BMC Med. Genomics 2010; 3: 48.

4 Nakamuta M, Fujino T, Yada R et al. Expression profiles of genes associated with viral entry in HCV-infected human liver. J. Med. Virol. 2011; 83: 921-7.

5 Reynolds GM, Harris HJ, Jennings A et al. Hepatitis C virus receptor expression in normal and diseased liver tissue. Hepatology 2008; 47: 418-27.

6 Zadori G, Gelley F, Torzsok P et al. Examination of claudin-1 expression in patients undergoing liver transplantation owing to hepatitis C virus cirrhosis. Transplant. Proc. 2011; 43: 1267-71.

7 Poenisch M, Bartenschlager R. New insights into structure and replication of the hepatitis $\mathrm{C}$ virus and clinical implications. Semin. Liver Dis. 2010; 30: 333-47.

8 Davidson-Moncada J, Papavasiliou FN, Tam W. MicroRNAs of the immune system: roles in inflammation and cancer. Ann. N. Y. Acad. Sci. 2010; 1183: 183-94.

9 Liu X, Wang T, Wakita T, Yang W. Systematic identification of microRNA and messenger RNA profiles in hepatitis $\mathrm{C}$ virus-infected human hepatoma cells. Virology 2010; 398: 57-67.

10 Neilson JR, Zheng GX, Burge CB, Sharp PA. Dynamic regulation of miRNA expression in ordered stages of cellular development. Genes Dev. 2007; 21: 578-89.

11 Jin X, Chen YP, Kong M, Zheng L, Yang YD, Li YM. Transition from hepatic steatosis to steatohepatitis: unique microRNA patterns and potential downstream functions and pathways. J. Gastroenterol. Hepatol. 2012; 27: 331-40.

12 Lendvai G, Kiss A, Kovalszky I, Schaff Z. [Alterations in microRNA expression patterns in liver diseases]. Orv. Hetil. 2010; 151: $1843-53$.

13 Morita K, Taketomi A, Shirabe K et al. Clinical significance and potential of hepatic microRNA-122 expression in hepatitis C. Liver Int. 2011; 31: 474-84.

14 Schickel R, Boyerinas B, Park SM, Peter ME. MicroRNAs: key players in the immune system, differentiation, tumorigenesis and cell death. Oncogene 2008; 27: 5959-74.

15 Asselah T, Bieche I, Sabbagh A et al. Gene expression and hepatitis C virus infection. Gut 2009; 58: 846-58.

16 Jopling CL, Schutz S, Sarnow P. Position-dependent function for a tandem microRNA miR-122-binding site located in the hepatitis C virus RNA genome. Cell Host Microbe 2008; 4: 77-85.

17 Pedersen IM, Cheng G, Wieland S et al. Interferon modulation of cellular microRNAs as an antiviral mechanism. Nature 2007; 449: 919-22.

18 Berkhout B. A balancing act: viruses and miRNAs. J. Formos. Med. Assoc. 2008; 107: 1-3.

19 Tombol Z, Szabo PM, Molnar V et al. Integrative molecular bioinformatics study of human adrenocortical tumors: microRNA, tissue-specific target prediction, and pathway analysis. Endocr. Relat. Cancer 2009; 16: 895-906.

20 Andersen CL, Jensen JL, Orntoft TF. Normalization of real-time quantitative reverse transcription-PCR data: a model-based variance estimation approach to identify genes suited for normalization, applied to bladder and colon cancer data sets. Cancer Res. 2004; 64: 5245-50. 
21 Nemes B, Sarvary E, Gerlei Z et al. [The recurrence of hepatitis C virus after liver transplantation]. Orv. Hetil. 2007; 148: 1971-9.

22 Berenguer M, Lopez-Labrador FX, Wright TL. Hepatitis C and liver transplantation. J. Hepatol. 2001; 35: 666-78.

23 Norman KL, Sarnow P. Hepatitis C virus' Achilles' heel-dependence on liver-specific microRNA miR-122. Cell Res. 2010; 20: 247-9.

24 Dai R, Ahmed SA. MicroRNA, a new paradigm for understanding immunoregulation, inflammation, and autoimmune diseases. Transl. Res. 2011; 157: 163-79.

25 Shenouda SK, Alahari SK. MicroRNA function in cancer: oncogene or a tumor suppressor? Cancer Metastasis Rev. 2009; 28: 369-78.

26 Chang J, Guo JT, Jiang D, Guo H, Taylor JM, Block TM. Liver-specific microRNA miR-122 enhances the replication of hepatitis C virus in nonhepatic cells. J. Virol. 2008; 82: 8215-23.

27 Henke JI, Goergen D, Zheng J et al. microRNA-122 stimulates translation of hepatitis C virus RNA. EMBO J. 2008; 27: 3300-10.

28 Randall G, Panis M, Cooper JD et al. Cellular cofactors affecting hepatitis C virus infection and replication. Proc. Natl Acad. Sci. U.S.A. 2007; 104: 12884-9.

29 Buhler S, Bartenschlager R. New targets for antiviral therapy of chronic hepatitis C. Liver Int. 2012; 32 (Suppl. 1): 9-16.

30 Wang XW, Heegaard NH, Orum H. MicroRNAs in liver disease. Gastroenterology 2012; 142: 1431-43.

31 Sarasin-Filipowicz M, Krol J, Markiewicz I, Heim MH, Filipowicz W. Decreased levels of microRNA miR-122 in individuals with hepatitis C responding poorly to interferon therapy. Nat. Med. 2009; 15: $31-3$.

32 Meng Z, Fu X, Chen X et al. miR-194 is a marker of hepatic epithelial cells and suppresses metastasis of liver cancer cells in mice. Hepatology 2010; 52: 2148-57.
33 Venugopal SK, Jiang J, Kim TH et al. Liver fibrosis causes downregulation of miRNA-150 and miRNA-194 in hepatic stellate cells, and their overexpression causes decreased stellate cell activation. Am. J. Physiol. Gastrointest. Liver Physiol. 2010; 298 : G101-6.

34 Jazbutyte V, Thum T. MicroRNA-21: from cancer to cardiovascular disease. Curr. Drug Targets 2010; 11: 926-35.

35 Wang Y, Lee CG. Role of miR-224 in hepatocellular carcinoma: a tool for possible therapeutic intervention? Epigenomics2011; 3: 235-43.

36 Scisciani C, Vossio S, Guerrieri F et al. Transcriptional regulation of miR-224 upregulated in human HCCs by NFkappaB inflammatory pathways. J. Hepatol. 2012; 56: 855-61.

37 Tardif KD, Waris G, Siddiqui A. Hepatitis C virus, ER stress, and oxidative stress. Trends Microbiol. 2005; 13: 159-63.

38 Pineau P, Volinia S, McJunkin K et al. miR-221 overexpression contributes to liver tumorigenesis. Proc. Natl Acad. Sci. U.S.A. 2010; 107: 264-9.

39 Zhang C, Han L, Zhang A et al. Global changes of mRNA expression reveals an increased activity of the interferon-induced signal transducer and activator of transcription (STAT) pathway by repression of miR-221/222 in glioblastoma U251 cells. Int. J. Oncol. 2010; 36: 1503-12.

40 Estrabaud E, Bieche I, Lapalus M et al. Hepatic miRNA signature associated with treatment response in chronic hepatitis $\mathrm{C}$. Hepatology 2011; 54 (Suppl. 1): 1344A.

41 Coulouarn C, Factor VM, Andersen JB, Durkin ME, Thorgeirsson SS. Loss of miR-122 expression in liver cancer correlates with suppression of the hepatic phenotype and gain of metastatic properties. Oncogene 2009; 28: 3526-36. 